\title{
Analysis PSNR of High Density Salt and Pepper Impulse Noise Using Median Filter
}

\author{
Sonali Malviya, Prof. Anshuj Jain \\ Department of Electronics \& Communication Engineering, \\ Scope College of Engineering, Bhopal, Madhya Pradesh, India
}

\begin{abstract}
In this paper a new method for the enhancement of gray scale images is introduced, when images are corrupted by fixed valued impulse noise (salt and pepper noise). The proposed methodology ensures a better output for low and medium density of fixed value impulse noise as compare to the other famous filters like Standard Median Filter (SMF), Decision Based Median Filter (DBMF) and Modified Decision Based Median Filter (MDBMF) etc. The main objective of the proposed method was to improve peak signal to noise ratio (PSNR), visual perception and reduction in blurring of image. The proposed algorithm replaced the noisy pixel by trimmed mean value. When previous pixel values, 0 's and $255^{\text {'s }}$ are present in the particular window and all the pixel values are
\end{abstract}

0 's and $255^{\circ} \mathrm{s}$ then the remaining noisy pixels are replaced by mean value. The gray-scale image of mandrill and Lena were tested via proposed method. The experimental result shows better peak signal to noise ratio (PSNR), mean square error (MSE) and mean absolute error (MAE) values with better visual and human perception.

KEY WORDS: Mean Square Error, Peak Signal to Noise Ratio. Pepper noise

\section{INTRODUCTION}

In the field of image processing, digital images very often get corrupted by several kinds of noise during the process of image acquisition. The basic reasons are malfunctioning of pixels in camera sensors, faulty memory locations in hardware, or transmission in a noisy channel [1]. In addition, these are also the main reasons responsible for generation of the impulse noise in digital world. In the field of image processing, digital images are mainly corrupted by the impulse noise [2]. There are two types of impulse noise, namely, the salt-and-pepper noise also known as the fixed valued impulse noise and the randomvalued impulse noise [3], [8]. Impulse noise is one the most severe noise which usually affects the images. Researchers are involved in the field of image denoising in order to find out effective method, capable of preserving the image details, reducing the noise of digital images and ensuring the quality of the image. Image quality measurement is analyzed by image parameters like peak to single noise ratio (PSNR), mean square error (MSE), image enhancement factor (IEF)[13], but in case of image processing one more thing of utmost importance is human perception [4], [9]. In this paper focus is kept upon salt and pepper noise. The salt and pepper noise corrupted pixels of image take either maximum or minimum pixel value Salt and pepper noise. Fixed valued impulse noise is producing two gray level values 0 and 255 . Random valued impulse noise will produce impulses whose gray level value lies within a predetermined range. The random value impulse noise is between 0 and 255. Generally the spatial domain filters have a detection stage which identifies the noisy and noise free pixels of the corrupted image, after that noise removal part removes the noise from the corrupted image under process while preserving the other important detail of image [5]. Initially standard median filter was popularly used, but later on switching based median filters came into existence which provides better results. Any other result oriented standard median filters are, weighted median filter, SDROM filter, centre weighted median filter, adaptive median filter, rank order median filter and many other improved filters. The performance of median filters also depends on the size of window of 
the filter. Larger window has the great noise suppression capability, but image details (edges, corners, fine lines) preservation is limited, while a smaller window preserves the details, but it will cause the reduction in noise suppression. Noise detection is a vital part of a filter, so it is necessary to detect whether the pixel is noise or noise free. However, further reduction in computational complexity is enviable.

\section{RELATIVE WORK}

Mean filter (M.F) In the 1998 Scott E Umbaugh, Computer Vision and Image Processing, Prentice Hall PTR, New Jersey, A mean filter acts on an image by smoothing it; that is, it reduces the intensity variation between adjacent pixels. The mean filter is nothing but a simple sliding window spatial filter that replaces the center value in the window with the average of all the neighboring pixel values including it. By doing this, it replaces pixels that are unrepresentative of their

surroundings. It is implemented with a convolution mask, which provides a result that is a weighted sum of the values of a pixel and its neighbors. It is also called a linear filter. The mask or kernel is a square. Often a $3 \times 3$ square kernel is used. If the coefficients of the mask sum up to one, then the average brightness of the image is not changed. If the coefficients sum to zero, the average brightness is lost, and it returns a dark image. The mean or average filter works on the shift-multiply-sum principle [12]. B. Adaptive median filter (AMF) In 2008, S.Saudia, Justin Varghese, Krishnan Nallaperumal, Santhosh.P.Mathew, Angelin J Robin, S.Kavitha, Proposes a new adaptive 2D spatial filter operator for the restoration of salt \& pepper impulse corrupted digital images name as --Salt \& Pepper Impulse Detection and Median based Regularization using Adaptive Median Filter\|, The Adaptive Impulse Filter effectively identifies the impulsive positions with a valid impulse noise detector and replaces them by a reliable signal determined from an appropriate neighborhood. Experimental results in terms of objective metrics and visual analysis show that the proposed algorithm performs better than many of the prominent median filtering techniques reported in terms of retaining the fidelity of even highly impulse corrupted images. High objectiveness and visual reliability is provided by the new restoration algorithm at lower quantum of impulse noise also. The Adaptive Median Filter (AMF) for salt \& pepper impulse noise removal that can give much acceptable and recognizable image restoration with better visual quality at all impulse noise levels than most other median filters which develop impulse patches in the output at higher impulse noise levels. Images restored by the proposed filter for Noise ratio at $95 \%$ restoration of the Proposed Filter with better objective metrics and fidelity at higher noise ratios is an improvement in the field of impulse restoration. The computational efficiency of the proposed filter is also significant at all impulse noise ratios. In 2009, Cheng Huang, Youlian Zhu, enhance the previous morphological filter and presented a -New MorphologicalFiltering Algorithm for Image Noise Reduction\|. Conventional morphological filter is disabling to effectively preserve image details while removing noises from an image. This algorithm of the self-adaptive median morphological filter is implemented as follows. First, the extreme value operation is displaced by the median operation in erosion and dilation. Then, the structuring element unit (SEU) is built based on the zero square matrix. Finally, the peak signal to noise ratio (PSNR) is used as the estimation function to select the size of the structuring element. Both the characteristics of morphological operations and the SEU determine the image processing effect. Following the increase of the noise density, the conventional morphological filtering algorithm and the median filtering algorithm become unavailable quickly. However, the proposed morphological filtering algorithm still has better effect in image noise reduction, especially in low SNR situations. Thus, the proposed algorithm is obviously superior to others [7]. C. Decision based algorithm (DBA) In 2009, S. Balasubramanian, S. Kalishwaran, R. Muthuraj, D. Ebenezer, V. Jayaraj presented -An Efficient Non-linear Cascade Filtering Algorithm for Removal of High Density Salt and Pepper Noise in Image and Video sequencell, in which an efficient non-linear cascade filter for the removal of high density salt and pepper noise in image and video is proposed. The proposed method consists of two stages to enhance the filtering. The first stage is the Decision based Median Filter (DMF), which is used to identify pixels likely to be contaminated by salt and pepper noise and replaces them by the median value. The second stage is the Unsymmetrical Trimmed Filter, either Mean Filter (UTMF) or Midpoint Filter (UTMP) which is used to trim the noisy pixels in an unsymmetrical manner and processes with the remaining pixels The basic idea is that, though the level of de-noising in the first stage is lesser at high noise densities, the second stage helps to increase the 
noise suppression. Hence, the proposed cascaded filter, as a whole is very suitable for low, medium as well as high noise densities even above $90 \%$. The existing non-linear filters such as Standard Median Filter (SMF), Adaptive Median Filter (AMF), Weighted Median Filter (WMF), Recursive Weighted Median Filter (RWMF) performs well only for low and medium noise densities. The recently proposed Decision Based Algorithm (DBA) shows better results up to $70 \%$ noise density and at high noise densities, the restored image quality is poor. The proposed algorithm shows better image and video quality in terms of visual appearance and quantitative measures. D. Modified dision based algorithm (MDBA) In 2009 an improved version of DBA is used to avoid streaks in images that usually occur in DBA due to repeated replacement of the noisy pixel with neighborhood pixels. In case of MDBA noisy pixels are replaced by the median of asymmetric trimmed output. Drawback of MDBA is that under high noise densities the pixels could be all 0 's or all $255^{\text {'s }}$ or a combination of both 0 and 255. Replacement with trimmed median value is not possible then. E. Decision based unsymmetrical trimmed mean filter (DBUTMF) In $2010 / \mathrm{K}$. Aiswarya, V. Jayaraj, and D. Ebenezer, proposed a new method for removal of high density salt and pepper noise (SNP) that is - - A new and efficient algorithm for the removal of high density salt and pepper noise in images and videos, $\|$ in Second Int. Conf. Computer Modeling and Simulation. To overcome the above drawback, Decision Based Algorithm (DBA) is proposed. In this, image is denoised by using a $3 \times 3$ window. If the processing pixel value is 0 or 255 it is processed or else it is left unchanged. At high noise density the median value will be 0 or 255 which is noisy. In such case, neighboring pixel is used for replacement. This repeated replacement of neighboring pixel produces streaking effect. In order to avoid this drawback, DecisionBased Unsymmetric Trimmed Median Filter (DBUTMF) is proposed [11], [10].

\section{PROPOSED ARCHITECTURE}

The proposed method deploys the enhancement by Modified Non-linear Filter (MNF) [1] algorithm. In this method first task is to detect the noisy pixels in the corrupted image. For detection of noisy pixels verifying the condition whether targeted pixel lies. If pixels are between maximum [255] and minimum [0] gray level values, then it is a noise free pixel, else pixel is said to be corrupted or noisy. Now we have processed only with the corrupted pixels to restore with noise free pixels. Further un-corrupted pixels are left unaffected. In the next steps we use Proposed Robust Mean filter (RMF) which is elucidated as follows. A. Algorithm Step 1: Initially in the very first step an image was taken and fixed valued impulses noise (Salt and Pepper noise) was applied on the image. Step 2: In the second it had been checked whether the pixels falls in between 0 to 255 ranges or not, consequently two cases were arises. $X(i, j)=$ $0<\mathrm{Y}(\mathrm{i}, \mathrm{j})<\mathrm{Y}(\mathrm{i}, \mathrm{j})$

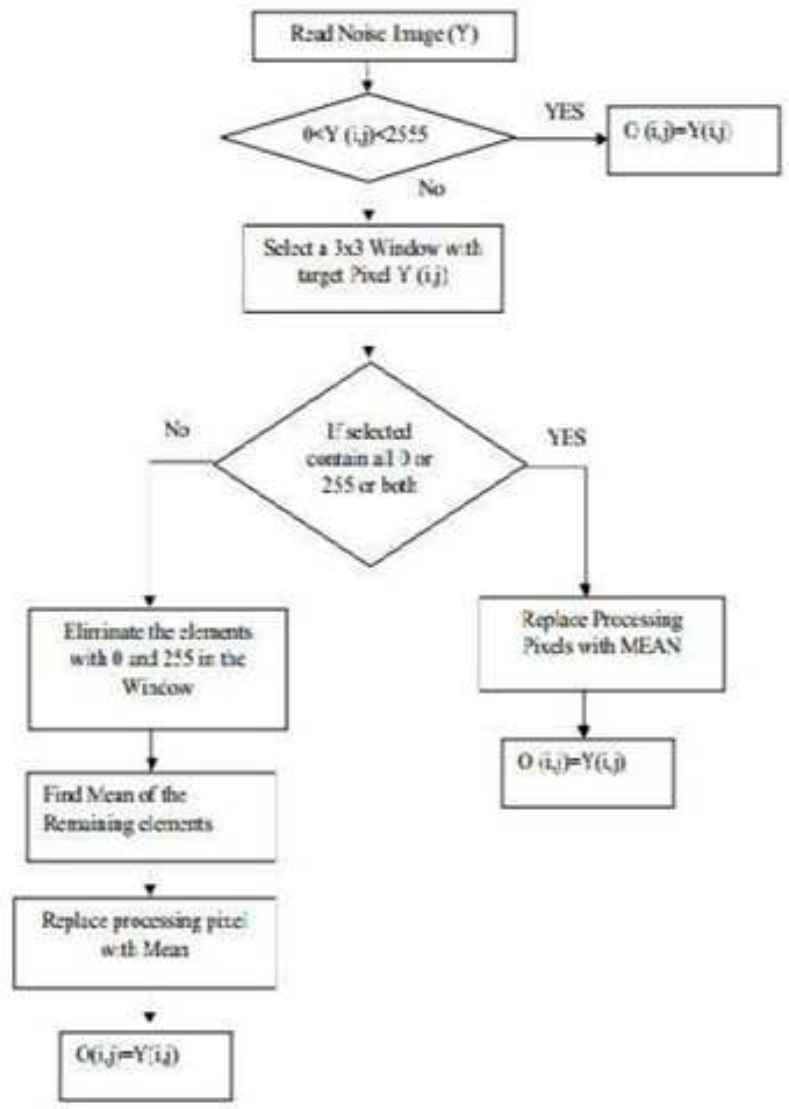

Figure 1: Flow-Chart diagram of Robust Mean Filter

\section{SIMULATION RESULT}

Any processing applied to an image may cause an important loss of information or quality.

Table 1: Comparison of MSE values of different filters for Lean image

\begin{tabular}{|c|c|c|c|c|c|}
\hline \multirow{2}{*}{$\begin{array}{l}\text { De-aoising } \\
\text { Metbods }\end{array}$} & \multicolumn{5}{|c|}{ Noise deasity } \\
\hline & $50 \%$ & $60 \%$ & $70 \%$ & $80 \%$ & $90 \%$ \\
\hline MIF & 2057,7 & 3919.5 & 6808.9 & 10071.1 & 14557.2 \\
\hline CWM & 32589 & 5408.5 & 8376.8 & 11300.0 & 152433 \\
\hline PSM & 650.25 & 1963.9 & 5048.5 & 9619.0 & 149139 \\
\hline IMF & 264.9 & 493.1 & 1422.6 & 4009.4 & 10305.7 \\
\hline SDROM & 23609 & 4396.2 & 7465.8 & 107914 & 14396.3 \\
\hline ACWM & 2153.1 & 4009.4 & 6967.5 & 100711 & 14557.2 \\
\hline ACWMIR & 540.8 & 1007.1 & 20562 & 4296.1 & 100711 \\
\hline TSMI & 3492.0 & 5930.3 & 9398.9 & 12678.8 & 163335 \\
\hline LEAM & 382.8 & 504.7 & 650.8 & 897.5 & 1267.8 \\
\hline $\begin{array}{l}\text { PROPOSED } \\
\text { METHOD }\end{array}$ & 39181 & 48203 & 66539 & 76397 & 877.16 \\
\hline
\end{tabular}


The PSNR is most commonly used as a measure of quality of reconstruction of loss compression codecs e.g., for image compression. The signal in this case is the original data, and the noise is the values introduced by impulsive noise. When comparing denoising results it is used as an approximation to human visibility of reconstruction quality, therefore in some cases one denoising results may appear to be closer to the original than another methods, even though it has a lower PSNR and a higher PSNR would normally indicate that the denoising method is of higher quality. We have to be extremely careful with the range of results; it is only comparably valid when it is used to compare results from the different denoising algorithms and same content.
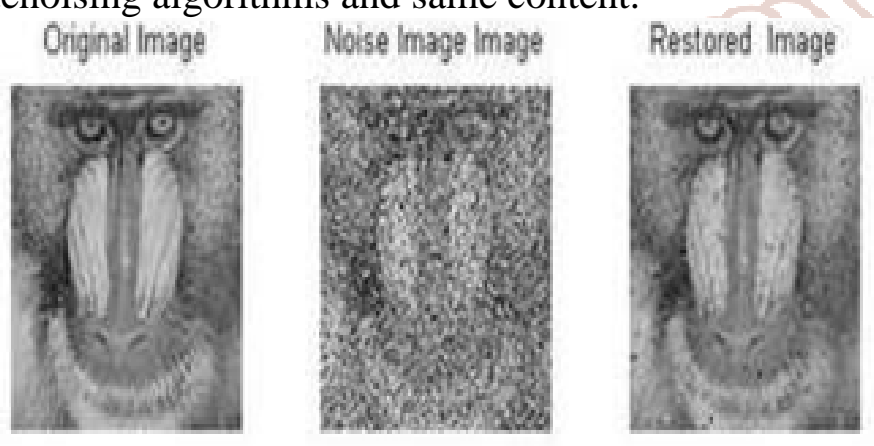

(b) 904 De-oodied lmaze

Figure 2: Experimental Results of Proposed Method for Baboon Image

\section{CONCLUSION}

The performance of the proposed algorithm has been tested at low, medium and high noise densities on gray scale images and also tested in real time images. Results reveal that the proposed filter exhibits better performance in comparison with $\mathrm{MF}, \mathrm{AMF}, \mathrm{DBA}$, MDBA, MDBUTMF, MNF filters in terms of higher PSNR. Indifference to AMF and other existing algorithms, the new algorithm uses a small $3 \times 3$ window having only eight neighbors of the corrupted pixel that have higher connection; this provides more edge information, more important to better edge preservation as well as more better Human \& visual perception. Infect at high noise density levels the new proposed algorithm gives better performance as compare with other existing de-noising filters. In the field of image processing we have not only improved the PSNR, MSE and MAE as well also require improving the visual quality for both types of images standard image and real time images.

\section{REFERENCES}

1. Raymond H. Chan, Chung-Wa Ho, and Mila Nikolova \| Salt-and-Pepper Noise Removal by Median-Type Noise Detectors and DetailPreserving Regularization\| IEEE TRANSACTIONS ON IMAGE PROCESSING, VOL. 14, NO. 10, OCTOBER 2005.

2. S. Esakkirajan, T. Veerakumar, Adabala N. Subramanyam and C. H. PremChand. - Removal of High Density Salt and Pepper Noise Through Modified Decision Based Unsymmetric Trimmed Median Filter.\| IEEE Signal Processing Letters, VOL. 18, NO. 5, MAY 2011.

3. P. E. Ng and K. K. Ma, -A switching median filter with boundary discriminative noise detection for extremely corrupted images, $\| I E E E$ Trans. Image Process., vol. 15, no. 6, pp. 1506-1516, Jun. 2006.

4. T. Sunilkumar, A. Srinivas, M. Eswae Reddy and Dr. G. Ramachandren Reddy - Removal of high density impulse noise through modified non-linear filter\| Journal of Theoretical and Applied Information Technology.. Vol. 47 No.2, 20th January 2013 ISSN: 1992-8645, E-ISSN: $1817-$ 3195.

5. S. Zhang and M. A. Karim, -A new impulse detector for switching median filters, IEEE Signal Process. Lett., vol. 9, no. 11, pp. 360-363, Nov. 2002.

6. Hossein Hosseini and Farokh Marvasti -Fast restoration of natural images corrupted by highdensity impulse noisell. Springer-EURASIP Journal on Image and Video Processing 2013, 2013:15.

7. H. Hwang and R. A. Hadded, -Adaptive median filter: New algorithms and results,\| IEEE Trans. Image Process., vol. 4, no. 4, pp. 499-502, Apr. 1995.

8. V. Jayaraj and D. Ebenezer, -A new switchingbased median filtering scheme and algorithm for removal of highdensity salt and pepper noise in image,\| EURASIP J. Adv. Signal Process., 2010.

9. T. A. Nodes and N.C. Gallagher, Jr., -The output distribution of median type filters, $\|$ IEEE Trans. Communication., 32(5): 532-541, 1984.

10. V. Jayaraj and D. Ebenezer, -A new switchingbased median filtering scheme and algorithm for removal of high density salt and pepper noise in 
International Journal of Trend in Scientific Research and Development (IJTSRD) ISSN: 2456-6470

removal of high-density salt and pepper noise in image,\| EURASIP J. Adv. Signal Process., 2010.

11. K. Aiswarya, V. Jayaraj, and D. Ebenezer, -A new and efficient algorithm for the removal of high density salt and pepper noise in images and videos, $\|$ in Second Int. Conf. Computer Modeling and Simulation, 2010, pp. 409-413.

12. J. Astola and P. Kuosmaneen, Fundamentals of Nonlinear Digital Filtering. Boca Raton, FL: CRC, 199J. Clerk Maxwell, A Treatise on Electricity and Magnetism, 3rd ed., vol. 2. Oxford: Clarendon, 1892, pp.68-73.

13. T. Sunilkumar, A. Srinivas, M. Eswar Reddy and Dr. G. Ramachandra Reddy, "Removal Of High Density Impulse Noise Through Modified NonLinear Filter='Journal of Theoretical and Applied Information Technology Vol. 47 No.2, 20th January 2013 ISSN: 1992-8645, E-ISSN: 18173195.

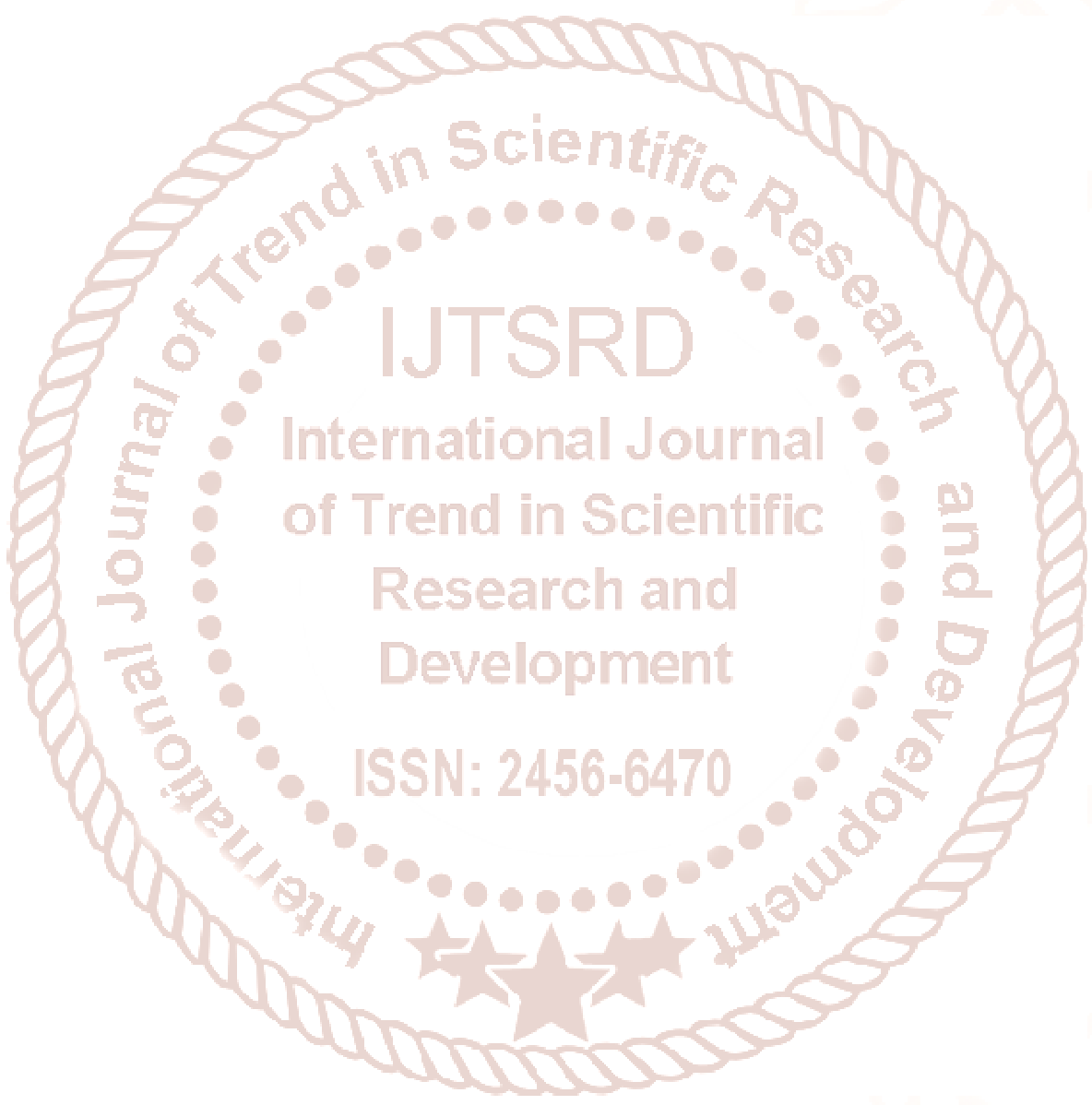

